\title{
Radiotherapy intensification for glioblastoma: enhancing the backbone of treatment
}

\author{
Vinai Gondi \\ Brain \& Spine Tumor Center, Northwestern Medicine Cancer Center Warrenville and Northwestern Medicine Proton Center, Warrenville, \\ IL, USA \\ Correspondence to: Vinai Gondi, MD. Northwestern Medicine Cancer Center Warrenville, 4405 Weaver Parkway, Warrenville, IL 60555, USA. \\ Email: vinai.gondi@nm.org.
}

\begin{abstract}
Given the impact of radiotherapy on survival outcomes for patients with glioblastoma (GBM), intensification of radiotherapy through dose-escalation and/or dose-per-fraction escalation has been an important area of ongoing investigation. Prior to the introduction of temozolomide, radiotherapy intensification beyond 60-Gy conventionally fractionated radiotherapy did not yield a survival benefit. With the emergence of temozolomide and its radio-sensitizing properties, as well as greater understanding of the patterns of first progression after 60-Gy radiotherapy, hypotheses regarding the impact of radiotherapy intensification have arisen. This article will discuss ongoing and future investigations of radiotherapy intensification in the modern temozolomide era of GBM management. These efforts have focused on better radiotherapy delivery techniques and/or improved tumor imaging to identify high-risk regions of progression.
\end{abstract}

Keywords: Glioblastoma (GBM); radiotherapy; intensity-modulated radiation therapy (IMRT); proton therapy; temozolomide

Submitted Jul 11, 2021. Accepted for publication Aug 13, 2021.

doi: $10.21037 /$ cco-21-109

View this article at: https://dx.doi.org/10.21037/cco-21-109

\section{Background}

Radiotherapy forms the backbone of survival-prolonging treatment for glioblastoma (GBM), an unfortunately incurable primary malignant brain tumor. Despite surgery, conventional radiotherapy, chemotherapy, and in some circumstances tumor-treating fields, the median survival for GBM remains poor at approximately $15-16$ months in contemporary series (1-3). Radiotherapy typically focuses on treatment of the surgical bed, MR-visible enhancing and non-enhancing tumor, and a margin of 1-2 $\mathrm{cm}$ of surrounding non-infiltrative tumor. The addition of temozolomide during and after radiotherapy enhances the therapeutic efficacy by prolonging time to tumor progression and increasing survival $(4,5)$.

Although radiotherapy-temozolomide combination has been shown to improve survival, the predominant pattern of radiographic failure remains local, within the parenchymal brain encompassing residual enhancing tumor and/or around the surgical bed and receiving the highest radiotherapy dose (i.e., $>57$ Gy or the $95 \%$ isodose line of prescribed $60 \mathrm{~Gy})(6,7)$. This may be due in part to the hypoxic microenvironment of GBM, which not only releases pro-angiogenic factors such as vascular endothelial growth factor (VEGF) (8), but also harbors stem cells that resist treatment and are putatively responsible for tumor regrowth (9).

\section{Radiotherapy intensification without chemotherapy}

Intensification of radiotherapy, through concomitant escalation of radiotherapy dose and dose-per-fraction, is an emerging approach to overcome hypoxia-related treatment resistance. Standard radiotherapy currently employs a total dose of $60 \mathrm{~Gy}$ in 30 fractions in 2-Gy fractions. This 
is based on historical dose-response analyses in the pretemozolomide era. Walker et al. reported on 420 patients treated on Brain Tumor Cooperative Group protocols and observed significant improvement in median survival from 28 to 42 weeks in the patients treated with doses of 50-60 Gy, compared to lower doses (10). Similarly, a Medical Research Council study of 443 patients also showed a significant survival advantage (median survival $12 \mathrm{vs}$. 9 months) in patients who received 60 Gy compared to those who received 45 Gy (11).

Radiotherapy intensification beyond 60 Gy has not demonstrated further survival benefit in prospective trials. Importantly, these trials were conducted either without chemotherapy or with nitrosurea-based chemotherapy and did not employ the current standard approach using temozolomide as a radiosensitizer. The Radiation Therapy Oncology Group (RTOG) and Eastern Cooperative Oncology Group (ECOG) observed no survival benefit with a $10-G y$ focal boost to $60-G y$ whole-brain irradiation (12). Intensity-modulated radiotherapy to a dose of 90 Gy for 34 patients with high-grade gliomas led to median survival of 11.7 months and 1- and 2-year survivals of $47.1 \%$ and $12.9 \%$, respectively, comparable to historical controls (13).

Hyperfractionated or accelerated radiotherapy regimens have also been explored as a means to intensify radiotherapy, using twice daily, three times daily, and even four times daily fractionation. RTOG 83-02 examined dose escalation using twice daily fractionation in patients with malignant gliomas and observed no survival differences between hyperfractionated regimens $64.8,72.0,76.8$, and 81.6 Gy given in 1.2-Gy fractions twice daily and accelerated hyperfractionated regimens of 48 and 54.4 Gy given in 1.6 Gy twice-daily fraction in combination with BCNU chemotherapy (14). A phase III trial observed no survival benefit of hyperfractionated radiotherapy to $72 \mathrm{~Gy}$ in 60 fractions of 1.2 Gy given twice daily compared to conventional radiotherapy to 60 Gy in 30 daily fractions (15).

Radiosurgery and brachytherapy have also been explored as approaches to radiotherapy intensification and failed to show survival benefit in the pre-temozolomide era. These negative trials have included the addition of a radiosurgical boost to 60-Gy radiotherapy plus adjuvant BCNU (16), the addition of a fractionated stereotactic radiotherapy boost to 50-Gy radiotherapy for a cumulative dose of 70 or $78 \mathrm{~Gy}$ (17), and the addition of a brachytherapy boost to 50-Gy radiotherapy (18).

More recently, a systematic review and meta-analysis of 22 prospective trials demonstrated progression- free and overall survival benefits to dose-escalated radiotherapy (defined as equivalent dose in 2-Gy fractions) $>60$ Gy versus 60 Gy standard-dose radiotherapy without chemotherapy (19). In analysis comparing 108 patients across 4 studies treated with dose-escalated radiotherapy without chemotherapy versus 1,001 patients across 6 studies treated with standard-dose radiotherapy without chemotherapy, 1-year OS was significantly higher $(46.3 \%$ vs. $23.4 \%, \mathrm{P}=0.02)$. In analysis comparing 91 patients across 3 studies treated with dose-escalated radiotherapy versus 422 patients across 3 studies treated with standard-dose radiotherapy, 1-year PFS was significantly higher (17.9\% vs. $5.3 \%, \mathrm{P}=0.02)$. Given the limited availability of MGMT promoter methylation status on these studies, OS and PFS differences could not be assessed. In spite of this limitation, this meta-analysis provides evidence in support of the use of dose-escalated radiotherapy in GBM patients unable to receive chemotherapy.

\section{Radiotherapy intensification with chemotherapy}

In the contemporary era of concurrent and adjuvant temozolomide, escalation of both dose and dose-perfraction using IMRT was tested in a prospective phase I/II study. The maximum tolerated dose with temozolomide was 75 Gy in 30 fractions (2.5 Gy per fraction). Median survival was 20.1 months, suggesting improved efficacy compared to other contemporary studies. Interestingly, the probability of in-field failure decreased in with increasing dose-escalation. These findings paralleled results from prior studies of radiotherapy intensification without chemotherapy, where patterns of failure could be altered and local control improved with radiotherapy dose escalation (20-22).

Based on the promising results of radiotherapy intensification in the setting of concurrent and adjuvant temozolomide, NRG Oncology BN001 was launched to test the hypothesis that radiotherapy intensification with temozolomide for newly diagnosed GBM prolongs survival. The trial has used the maximum tolerated dose of $75 \mathrm{~Gy}$ in 30 fractions, delivered to the surgical bed and residual enhancing tumor plus a volumetric margin of $5 \mathrm{~mm}$, with differential dose-painting of $50 \mathrm{~Gy}$ in 30 fractions to non-enhancing tumor plus a $2-\mathrm{cm}$ volumetric margin, with concomitant and adjuvant temozolomide. The primary endpoint was overall survival. As per criteria of the preceding phase I/II trial, the trial was limited to patients with a surgical cavity of $5 \mathrm{~cm}$ or less.

The trial was designed as two randomized phase II trials 
running simultaneously. All arms of both cohorts received concurrent and adjuvant temozolomide. In the intensitymodulated radiation therapy (IMRT) cohort, patients were randomized to 75 Gy IMRT vs. 60 Gy IMRT. In the proton therapy cohort, patients were randomized to 75 Gy proton therapy $v s$. 60 Gy IMRT. The trial was developed using an instrumental variable analysis study design, wherein if the control arms of each cohort were statistically comparable, then the investigational arms of each cohort (75-Gy proton therapy versus 75-Gy IMRT) would be compared statistically for the primary endpoint. The trial was also designed as a signal-seeking study, using an inflated onesided alpha of 0.15 for statistical significance to prompt a validation phase III study. Given the widespread availability of IMRT relative to proton therapy, the IMRT cohort completed accrual in July 2018, while the proton therapy cohort continues to enroll and is slated to complete accrual in early 2022.

Preliminary results of the IMRT cohort were reported at the 2020 Annual Meeting of the American Society for Radiation Oncology (ASTRO) (23). From October 2014 to July 2018, 229 patients were eligible and randomized. Median age was 62 years; $58 \%$ were MGMT unmethylated; $75 \%$ were RPA class IV. Treatment arms did not differ in baseline characteristics or grade $\geq 3$ toxicity. Median survival was 18.7 months after 75-Gy IMRT versus 16.3 months after 60 -Gy IMRT. Though the survival curves were non-overlapping in favor of 75-Gy IMRT, the difference between treatment arms did not reach statistical significance for testing in phase III setting $(\mathrm{P}=0.19$ vs. $a$ priori $\mathrm{P}<0.15$ for statistical significance). At 30 months, $30.4 \%(95 \%$ CI: $21.8,39.1)$ on $75-$ Gy IMRT arm, and $21.6 \%$ (95\% CI: $12.7,30.6)$ on $60-$ Gy IMRT arm were alive.

Proton therapy for GBM is associated with a significant reduction in integral dose, which has been shown to prevent radiotherapy-induced circulating lymphopenia (24). Emerging data suggest that the circulating lymphocyte compartment is influenced by this integral dose and putatively represents a biologically relevant normal tissue compartment. In the pretemozolomide era, radiotherapy for high-grade glioma have demonstrated a significant reduction in CD4 counts over the course of treatment. Specifically, after 6 weeks of radiotherapy, $47 \%$ of patients had CD 4 counts $<300$ cells $/ \mathrm{mm}^{3}$ and $26 \%$ had CD 4 counts $<200$ cells $/ \mathrm{mm}^{3}(25)$. In a subsequent prospective multicenter observational trial of high-grade glioma patients treated with standard chemoradiotherapy, $40 \%$ of patients had CD 4 counts $<200$ cells $/ \mathrm{mm}^{3}$ by 2 months after initiating therapy (26). Importantly, after adjusting for known prognostic factors, patients with CD4 counts $<200$ cells $/ \mathrm{mm}^{3}$ had significantly inferior median survival as compared to those with higher CD4 counts (13.1 vs. 19.7 months, $\mathrm{P}=0.002$ ). Interestingly, the cause of death was attributable to early tumor progression, and not to opportunistic infections as was the original hypothesis. Thus, these findings highlight the putative importance of radiosensitive circulating CD4 lymphocytes on tumor control and survival, implicating an immunologic mechanism.

Given evidence that proton therapy prevents radiotherapy-induced circulating lymphopenia, and that circulating lymphopenia impacts survival outcomes in GBM patients, accrual to the cohort proton therapy cohort of NRG BN001 is encouraged, as proton therapy is hypothesized to provide an additional survival benefit beyond that potentially provided by radiotherapy intensification. Additionally, translational analyses of centrally submitted surgical specimens are planned to investigate biologic subtypes of GBM that may differentially benefit from radiotherapy dose-intensification.

Another area of investigation for radiotherapy intensification is the use of functional imaging to guide the target region for radiotherapy dose-escalation. Positron emission tomography (PET) imaging using L-3,4-dihydroxy-6- ${ }^{18}$ Ffluoro-phenyl-alanine $\left({ }^{18} \mathrm{~F}\right.$-DOPA). ${ }^{18} \mathrm{~F}$-DOPA is an amino acid that biochemically resembles natural L-DOPA, a precursor of the neurotransmitters dopamine, norepinephrine and epinephrine. Due to upregulation of amino acid transport in malignant tissues and thereby increased amino acid uptake in brain tumors compared to normal brain, ${ }^{18} \mathrm{~F}$-DOPA-PET has been observed to be sensitive and specific in identifying biologically aggressive and residual occult glioma beyond regions of MR-based contrast-enhancement (27).

In a single-institution phase II trial of ${ }^{18} \mathrm{~F}$-DOPA-PETguided radiotherapy dose-escalation (28), a dose-painting technique in 30 fractions was used to treat GBM patients to 51 Gy to the MR-defined surgical cavity, residual contrast enhancement and surrounding FLAIR abnormality, ${ }^{18}$ F-DOPA-PET-defined regions of lower-density and higher-density tumor infiltration, and a $1 \mathrm{~cm}$ volumetric margin; 60 Gy to the MR-defined surgical cavity and residual contrast enhancement, ${ }^{18} \mathrm{~F}$-DOPA-PET-defined regions of higher-density tumor infiltration, and a $1 \mathrm{~cm}$ volumetric margin; and, 76 Gy to the MR-defined surgical cavity and residual contrast enhancement and ${ }^{18} \mathrm{~F}$-DOPAPET-defined higher-density tumor infiltration. Treatment 
was delivered with concomitant and adjuvant temozolomide for 6 cycles. ${ }^{18} \mathrm{~F}$-DOPA-PET-defined regions of lowerversus higher-density tumor infiltration were based on computations of ratios of SUV-max of the tumor to SUVmean of the contralateral brain tissue $(\mathrm{T} / \mathrm{N})$. Regions of $\mathrm{T} / \mathrm{N}$ of 1.2 to 2.0 were defined as lower-density tumor infiltration; regions of $\mathrm{T} / \mathrm{N}$ of $>2.0$ were defined as higherdensity tumor infiltration.

On this trial, 75 evaluable patients were enrolled and treated with ${ }^{18} \mathrm{~F}$-DOPA-PET-guided radiotherapy doseintensification. Median PFS was longer than historical controls (8.75 vs. 6.6 months; $\mathrm{P}=0.017$ ), but the OS difference though numerically longer was not statistically significant (16.0 vs. 13.5 months, $\mathrm{P}=0.13$ ). Amongst MGMT promoter methylated patients, ${ }^{18} \mathrm{~F}$-DOPA-PET-guided radiotherapy dose-intensification was associated with longer OS compared to historical controls (35.5 vs. 23.3 months, $\mathrm{P}=0.049)$. Amongst MGMT promoter unmethylated patients, the OS difference though numerically longer was not statistically significant (16.0 vs. 13.5 months, $\mathrm{P}=0.13$ ). Following ${ }^{18} \mathrm{~F}$-DOPA-PET-guided radiotherapy doseintensification, 10 patients developed grade 3 CNS necrosis. Of 8 patients treated with bevacizumab for grade $3 \mathrm{CNS}$ necrosis, 7 patients developed improvement in neurologic symptoms to grade 2 or less necrosis.

The primary endpoint on this single-arm phase II trial was the proportion of MGMT promoter unmethylated patients who were free of progression or death by 6 months (PFS6). Following ${ }^{18} \mathrm{~F}$-DOPA-PET-guided radiotherapy dose-intensification for MGMT promoter unmethylated patients, 31 of 39 patients (79.5\%, 95\% CI: 63.1-90.1\%) were alive and free of progression, which was significantly higher than the PFS6 rate of 54.3\% (95\% CI: 44.5-66.3\%) from the historical control. The study team concluded that meeting this PFS6 threshold permitted consideration of further testing of ${ }^{18} \mathrm{~F}$-DOPA-PET-guided radiotherapy dose-intensification for MGMT promoter unmethylated patients, even though an OS difference was not observed in this subgroup.

\section{Conclusions}

Given the importance of increasing radiotherapy dose to $60 \mathrm{~Gy}$ in delaying progression and prolonging survival in GBM, radiotherapy intensification beyond standard 60 Gy is an ongoing and critical area of investigation. In patients not eligible to receive radio-sensitizing chemotherapy, a recently published meta-analysis supports an overall survival and progression-free survival benefit to radiotherapy intensification in excess of standard equivalent dose in 2-Gy fractions of $60 \mathrm{~Gy}$. In patients eligible to receive radiosensitizing chemotherapy, a recently presented randomized phase II trial observed a numerical but not statistically significant survival benefit following IMRT doseintensification. Opportunities to enhance survival further include the use of proton therapy to minimize effect of integral dose on circulating lymphopenia and/or the use of functional ${ }^{18} \mathrm{~F}$-DOPA-PET to better target high-risk tumor regions with radiotherapy intensification.

\section{Acknowledgments}

Funding: None.

\section{Footnote}

Provenance and Peer Review: This article was commissioned by the Guest Editor (Rimas V. Lukas) for the series "The Evolving Landscape of the Management of Glioblastoma" published in Chinese Clinical Oncology. The article has undergone external peer review.

Conflicts of Interest: The author has completed the ICMJE uniform disclosure form (available at http://dx.doi. org/10.21037/cco-21-109). The series "The Evolving Landscape of the Management of Glioblastoma" was commissioned by the editorial office without any funding or sponsorship. Dr. VG discloses honoraria from UpToDate outside the submitted work. The author has no other conflicts of interest to declare.

Ethical Statement: The author is accountable for all aspects of the work in ensuring that questions related to the accuracy or integrity of any part of the work are appropriately investigated and resolved.

Open Access Statement: This is an Open Access article distributed in accordance with the Creative Commons Attribution-NonCommercial-NoDerivs 4.0 International License (CC BY-NC-ND 4.0), which permits the noncommercial replication and distribution of the article with the strict proviso that no changes or edits are made and the original work is properly cited (including links to both the formal publication through the relevant DOI and the license). See: https://creativecommons.org/licenses/by-nc$\mathrm{nd} / 4.0 /$. 


\section{References}

1. Grossman SA, Ye X, Piantadosi S, et al. Survival of patients with newly diagnosed glioblastoma treated with radiation and temozolomide in research studies in the United States. Clin Cancer Res 2010;16:2443-9.

2. Gilbert MR, Wang M, Aldape KD, et al. RTOG 0525: A randomized phase III trial comparing standard adjuvant temozolomide with a dose-dense schedule in newly diagnosed glioblastoma. J Clin Oncol 2011;29:abstr 2006.

3. Stupp R, Taillibert S, Kanner A, et al. Effect of TumorTreating Fields Plus Maintenance Temozolomide vs Maintenance Temozolomide Alone on Survival in Patients With Glioblastoma: A Randomized Clinical Trial. JAMA 2017;318:2306-16.

4. Stupp R, Mason WP, van den Bent MJ, et al. Radiotherapy plus concomitant and adjuvant temozolomide for glioblastoma. N Engl J Med 2005;352:987-96.

5. Stupp R, Hegi ME, Mason WP, et al. Effects of radiotherapy with concomitant and adjuvant temozolomide versus radiotherapy alone on survival in glioblastoma in a randomised phase III study: 5-year analysis of the EORTC-NCIC trial. Lancet Oncol 2009;10:459-66.

6. Chan JL, Lee SW, Fraass BA, et al. Survival and failure patterns of high-grade gliomas after three-dimensional conformal radiotherapy. J Clin Oncol 2002;20:1635-42.

7. Milano MT, Okunieff P, Donatello RS, et al. Patterns and timing of recurrence after temozolomide-based chemoradiation for glioblastoma. Int J Radiat Oncol Biol Phys 2010;78:1147-55.

8. Rong Y, Hu F, Huang R, et al. Early growth response gene-1 regulates hypoxia-induced expression of tissue factor in glioblastoma multiforme through hypoxiainducible factor-1-independent mechanisms. Cancer Res 2006;66:7067-74.

9. Seidel S, Garvalov BK, Wirta V, et al. A hypoxic niche regulates glioblastoma stem cells through hypoxia inducible factor 2 alpha. Brain 2010;133:983-95.

10. Walker MD, Strike TA, Sheline GE. An analysis of doseeffect relationship in the radiotherapy of malignant gliomas. Int J Radiat Oncol Biol Phys 1979;5:1725-31.

11. Bleehen NM, Stenning SP. A Medical Research Council trial of two radiotherapy doses in the treatment of grades 3 and 4 astrocytoma. The Medical Research Council Brain Tumour Working Party. Br J Cancer 1991;64:769-74.

12. Nelson DF, Diener-West M, Horton J, et al. Combined modality approach to treatment of malignant gliomas-re-evaluation of RTOG 7401/ECOG 1374 with long- term follow-up: a joint study of the Radiation Therapy Oncology Group and the Eastern Cooperative Oncology Group. NCI Monogr 1988;(6):279-84.

13. Shin KH, Urtasun RC, Fulton D, et al. Multiple daily fractionated radiation therapy and misonidazole in the management of malignant astrocytoma. A preliminary report. Cancer 1985;56:758-60.

14. Werner-Wasik M, Scott CB, Nelson DF, et al. Final report of a phase I/II trial of hyperfractionated and accelerated hyperfractionated radiation therapy with carmustine for adults with supratentorial malignant gliomas. Radiation Therapy Oncology Group Study 83-02. Cancer 1996;77:1535-43.

15. Scott C, Curran W, Yung WK, et al. Long term results of RTOG 9006: A randomized trial of hyperfractionated radiotherapy (RT) to 72.0 Gy and carmustine vs. standard RT and carmustine for malignant glioma patients with emphasis on anaplastic astrocytoma (AA) patients. Proc Am Soc Clin Oncol 1998;16:384.

16. Souhami L, Seiferheld W, Brachman D, et al. Randomized comparison of stereotactic radiosurgery followed by conventional radiotherapy with carmustine to conventional radiotherapy with carmustine for patients with glioblastoma multiforme: report of Radiation Therapy Oncology Group 93-05 protocol. Int J Radiat Oncol Biol Phys 2004;60:853-60.

17. Cardinale R, Won M, Choucair A, et al. A phase II trial of accelerated radiotherapy using weekly stereotactic conformal boost for supratentorial glioblastoma multiforme: RTOG 0023. Int J Radiat Oncol Biol Phys 2006;65:1422-8.

18. Laperriere NJ, Leung PM, McKenzie S, et al. Randomized study of brachytherapy in the initial management of patients with malignant astrocytoma. Int J Radiat Oncol Biol Phys 1998;41:1005-11.

19. Singh R, Lehrer EJ, Wang M, et al. Dose Escalated Radiation Therapy for Glioblastoma Multiforme: An International Systematic Review and Meta-Analysis of 22 Prospective Trials. Int J Radiat Oncol Biol Phys 2021. [Epub ahead of print]. doi: 10.1016/j.ijrobp.2021.05.001.

20. Fitzek MM, Thornton AF, Rabinov JD, et al. Accelerated fractionated proton/photon irradiation to 90 cobalt gray equivalent for glioblastoma multiforme: results of a phase II prospective trial. J Neurosurg 1999;91:251-60.

21. Nakagawa K, Aoki Y, Fujimaki T, et al. High-dose conformal radiotherapy influenced the pattern of failure but did not improve survival in glioblastoma multiforme. Int J Radiat Oncol Biol Phys 1998;40:1141-9. 
22. Tanaka M, Ino Y, Nakagawa K, et al. High-dose conformal radiotherapy for supratentorial malignant glioma: a historical comparison. Lancet Oncol 2005;6:953-60.

23. Gondi V, Pugh S, Tsien C, et al. Radiotherapy doseintensification using intensity-modulated radiotherapy versus standard-dose radiotherapy with temozolomide in newly diagnosed glioblastoma: Preliminary results of NRG Oncology CC001. Int J Radiat Oncol Biol Phys 2020;108:S22-S23.

24. Mohan R, Liu AY, Brown PD, et al. Proton therapy reduces the likelihood of high-grade radiationinduced lymphopenia in glioblastoma patients: phase II randomized study of protons vs photons. Neuro Oncol 2021;23:284-94.

25. Hughes MA, Parisi M, Grossman S, et al. Primary brain

Cite this article as: Gondi V. Radiotherapy intensification for glioblastoma: enhancing the backbone of treatment. Chin Clin Oncol 2021;10(4):39. doi: 10.21037/cco-21-109 tumors treated with steroids and radiotherapy: low CD4 counts and risk of infection. Int J Radiat Oncol Biol Phys 2005;62:1423-6.

26. Grossman SA, Ye X, Lesser G, et al. Immunosuppression in patients with high-grade gliomas treated with radiation and temozolomide. Clin Cancer Res 2011;17:5473-80.

27. Pafundi DH, Laack NN, Youland RS, et al. Biopsy validation of 18F-DOPA PET and biodistribution in gliomas for neurosurgical planning and radiotherapy target delineation: results of a prospective pilot study. Neuro Oncol 2013;15:1058-67.

28. Laack NN, Pafundi D, Anderson SK, et al. Initial Results of a Phase 2 Trial of 18F-DOPA PET-Guided DoseEscalated Radiation Therapy for Glioblastoma. Int J Radiat Oncol Biol Phys 2021;110:1383-95. 